Ann. Biol. anim. Bioch. Biophys., 1971, 11 (4), 669-679.

\title{
ÉVOLUTION POSTNATALE DE LA QUANTITÉ D'EAU ET DE LIPIDES DU CORPS ET DU GRAND PSOAS CHEZ LE LAPIN
}

\author{
P, VIGNERON, R. BARON et L. DAUZIER \\ avec la collaboration technique de $\mathrm{C}$. Bressot \\ Station de Physiologie animale, I. N. R. A., \\ École nationale supérieure agronomique, \\ Place Viala, 34 - Montpellier
}

\section{RÉSUMÉ}

L'évolution postnatale de la composition biochimique du corps et du muscle Grand Psoas a été étudiée chez des lapins mâles et femelles.

Chez des animaux pris depuis la naissance jusqu'à plus d'un an, les teneurs en eau et en lipides passent respectivement de $79 \mathrm{p}$. Ioo à $72 \mathrm{p}$. Ioo et de $7 \mathrm{p}$. Ioo à $15 \mathrm{p}$. Ioo dans le muscle, de $76 \mathrm{p}$. roo à $50 \mathrm{p}$. Ioo et de ro $\mathrm{p}$. roo à $4^{\circ} \mathrm{p}$. roo pour l'organisme entier.

La croissance relative de la quantité de lipides et d'eau totale de l'organisme a été étudiée à l'aide de la relation d'allométrie. Dans les deux sexes, après une période d'isométrie, on note un changement d'allométrie silmultané et en sens inverse, l'allométrie des lipides devenant majorante, et celle de l'eau minorante.

La quantité (eau + lipides) évolue de façon isométrique à celle du corps pendant toute la vie postnatale, et de façon identique chez les mâles et les femelles.

Il est donc possible, connaissant la quantité d'eau et le poids vif de l'organisme, de déterminer la quantité de lipides, quel que soit l'âge ou le poids du Lapin.

\section{INTRODUCTION}

$\mathrm{Au}$ cours de la croissance l'organisme montre une déshydratation importante alors que le pourcentage de lipides s'accroît. Ceci est connu depuis longtemps (VoN BEZOL,D, I857; cité par MoulToN, I923) et a été confirmé par de nombreux auteurs, parmi lesquels ALLEN et al. (I959), FRIIS-HANSEN (I955) chez l'Homme ; EILLENBerger (I950), Moulton (I923) chez les bovins; Panaretto (I963), Panaretto et TILL (I963) chez les ovins et les caprins ; SPRAY et WIDdowson (I950) chez le Porc, le Lapin, le Chat, le Rat ; CHEEk et Hort (I963) chez la Souris. 
Les études sur les variations de composition des tissus et des organes sont moins nombreuses. Il semble que la composition du muscle varie peu avec l'âge chez la plupart des mammifères. En particulier les teneurs en lipides totaux et en eau totale intracellulaire ne présentent que de faibles variations susceptibles pourtant de rendre compte, du moins pendant la plus grande partie de la croissance postnatale, des changements de concentrations des diverses catégories de protéines musculaires, rapportés dans la littérature (GoRdon et al., I966 ; IvANov, I967 ; BARON et VIGNERON, 1970). Les variations observées chez 1'Homme seraient plus importantes (HELANDER, I959).

Il y aurait donc une différence importante entre 1'évolution de la composition de l'organisme considéré dans sa totalité et celle de la composition du tissu musculaire. Aussi nous a-t-il semblé intéressant d'étudier simultanément, chez une espèce domestique, l'évolution de la composition corporelle globale, et celle d'un muscle squelettique.

\section{MATÉRIEL ET MÉTHODES}

\section{I. - Matériel animal}

Les études ont porté sur une souche de lapins communs élevés au laboratoire et dont on connaît la croissance globale et relative (CANTIER et al., I969 ; BARON et al., 1970 ; VezinHeT et al., 197I).

Les animaux sont sevrés à 28 jours, et dès ce moment reçoivent à volonté des aliments complets granulés du commerce, contenant I $9 \mathrm{p}$. Ioo de matière azotée totale et $\mathrm{r}_{4} \mathrm{p}$. Ioo de cellulose.

Vingt-cinq mâles et 28 femelles ont été sacrifiés à des âges divers choisis de telle façon que les données soient plus nombreuses lorsque la vitesse de croissance est la plus rapide. En outre, nous avons sacrifié à roo jours une femelle ayant eu une croissance nettement retardée par rapport à la moyenne (2 I $5 \mathrm{~g}$ au lieu de $2700 \mathrm{~g}$ environ).

\section{TABLEAU I}

Répartition selon le sexe du nombre et du poids moyen des animaux abattus à chaque stade

\begin{tabular}{|c|c|c|c|c|}
\hline \multirow[t]{2}{*}{ Age } & \multicolumn{2}{|r|}{ Mâles } & \multicolumn{2}{|r|}{ Femelles } \\
\hline & $n$ & $\begin{array}{c}\text { Poids moyen } \\
\text { (g) }\end{array}$ & $n$ & $\begin{array}{l}\text { Poids moyen } \\
\text { (g) }\end{array}$ \\
\hline Naissance $\ldots \ldots \ldots \ldots \ldots$ & 4 & 55,3 & 4 & 48,7 \\
\hline 2 semaines $\ldots \ldots \ldots \ldots \ldots \ldots$ & 8 & 245 & 6 & 230 \\
\hline 4 semtaines $\ldots \ldots \ldots \ldots \ldots$ & 4 & 635 & 4 & 657 \\
\hline 7 semaines $\ldots \ldots \ldots \ldots \ldots$ & 2 & 1530 & 2 & 1462 \\
\hline 10 semaines $\ldots \ldots \ldots \ldots \ldots$ & 2 & 2027 & 2 & 2015 \\
\hline 16 semaines . ............ & 2 & 2900 & 2 & 2995 \\
\hline 26 semaines $\ldots \ldots \ldots \ldots \ldots$. & 1 & 4100 & 2 & 4295 \\
\hline 11 mois $\ldots \ldots \ldots \ldots \ldots \ldots$ & 2 & 4400 & 2 & 6250 et 4890 \\
\hline 1 an 2 mois $\ldots \ldots \ldots \ldots \ldots$ & & & 3 & 5600 \\
\hline 2 ans 6 mois . ............. & & & 1 & 5525 \\
\hline
\end{tabular}




\section{2. - Méthode d'analyse chimique}

Après étourdissement les animaux subissent une saignée aussi complète que possible. Le contenu du tractus digestif est prélevé et soumis à dessiccation sous vide à $60^{\circ} \mathrm{C}$ pendant 72 heures. Les muscles Grand Psoas sont désinsérés, introduits dans des flacons hermétiquement fermés et conservés à $-25^{\circ} \mathrm{C}$ dans un congélateur. Le reste de l'organisme, congelé au préalable à $-25^{\circ} \mathrm{C}$ est broyé à plusieurs reprises dans un hachoir à viandes refroidi (Scharfen) en utilisant des grilles de plus en plus fines. Le hachis obtenu est soigneusement homogénéisé, et des fractions de I à 3 grammes sont prélevées pour subir soit une dessiccation dans les mêmes conditions que le contenu digestif, soit un dosage de lipides totaux selon la technique de Folch (I957). Dans ce cas, les échantillons sont au préalable broyés plus finement, en présence d'eau, à l'aide d'un broyeur-homogénéiseur Ultra-Turrax.

Les mêmes analyses sont pratiquées sur les échantillons de muscle.

La quantité d'eau contenue dans le sang recueilli à la saignée a été calculée, sachant que chez le Lapin le sang contient 81,7 p. Ioo d'eau (DukEs, 1947).

Pour chaque animal nous avons disposé des données suivantes qui nous ont permis une analyse mathématique :

- Poids vif ;

- Poids vide (poids vif diminué de celui du contenu du tractus digestif et du sang) ;

- Poids du sang de saignée (contenant $8 \mathrm{I}, 7$ p. Ioo d'eau) ;

- Poids du contenu du tractus digestif et quantité d'eau retenue dans ce compartiment ;

- Poids du Psoas ;

- Eau du Psoas;

- Lipides du Psoas ;

- Eau totale;

- Lipides totaux.

Il n'a été tenu compte, dans les lipides totaux, ni des lipides sanguins, ni des lipides du contenu des réservoirs digestifs.

\section{3. - Analyse des résultats}

a) La velation d'allométrie.

La fonction puissance $y=k x^{a}$ (I) a été introduite par HuXLEY (I932) et TeIssier (I934) pour décrire la croissance relative de deux parties $y$ et $x$ d'un organisme.

La relation d'allométrie, d'utilisation aisée (CANTrER et al., I969) a déjà servi à divers auteurs dans l'étude de l'évolution de constituants chimiques au cours de la croissance (NEEDHAM, 1933; GuILlAUME et al., 1966, etc.).

L'interprétation physiologique du coefficient d'allométrie est classique : c'est le rapport des vitesses spécifiques de croissance

$$
\frac{\mathrm{d} y}{y \mathrm{dt}} \int \frac{\mathrm{d} x}{x \mathrm{dt}}
$$

b) Choix de la variable de référence. Méthode d'ajustement.

Nous avons choisi le poids vif comme variable de référence. Le choix du poids vide n'aurait apporté aucune amélioration puisque le poids vif et le poids vide sont liés linéairement au cours de la croissance (PRUD'HoN et al., I969). De plus l'usage du poids vif est d'un grand intérêt pratique dans ce type de travail du fait des applications éventuelles possibles lors d'estimations de composition corporelle in vivo.

Plusieurs méthodes sont utilisées pour le calcul des coefficients de la relation d'allométrie rendue linéaire par transformation logarithmique :

$$
\begin{aligned}
\log y & =b+a \log x \\
b & =\log k .
\end{aligned}
$$

Ces diverses méthodes ont été comparées par KERmack et Haldane (1950).

Dans le cas présent, compte tenu de la valeur très élevée des coefficients de corrélation entre $\log x$ et $\log y$, de l'ordre de 0,99 pour les divers composants étudiés, nous avons opté pour la méthode des moindres carrés, une analyse préalable nous ayant d'ailleurs montré que le calcul de l'axe majeur ou de l'axe majeur réduit n'offrait pas d'avantage particulier.

De même, pour l'étude du changement d'allométrie de l'eau totale et des lipides totaux de 
l'organisme, nous avions la possibilité d'utiliser soit une régression logarithmique polynomiale (Baron et Vigneron, 1970), soit la méthode préconisée par Rouvier (in : Cantier et al., I969), qui consiste à rechercher la variance résiduelle minimale. Nous avons choisi cette dernière, puisque les variations $\frac{\mathrm{d} y}{y} / \frac{\mathrm{d} x}{x}$ ne sont pas progressives, et qu'on observe un changement d'allométrie au sens strict.

En ce qui concerne la variable (eau + lipides totaux), l'ajustement est strictement linéaire, et les résultats concordent, quelle que soit la méthode choisie.

\section{RÉSULTATS}

Dans le tableau 2 sont rapportés les paramètres des droites d'allométrie calculées pour les divers constituants.

L'évolution de la teneur en eau et en lipides du corps et du muscle Grand Psoas est représentée figure 3 .

\section{I. - Évolution de l'eau et des lipides totaux corporels}

Au cours de la croissance postnatale, une augmentation de la quantité d'eau contenue dans l'organisme accompagne toujours un gain pondéral global mais selon

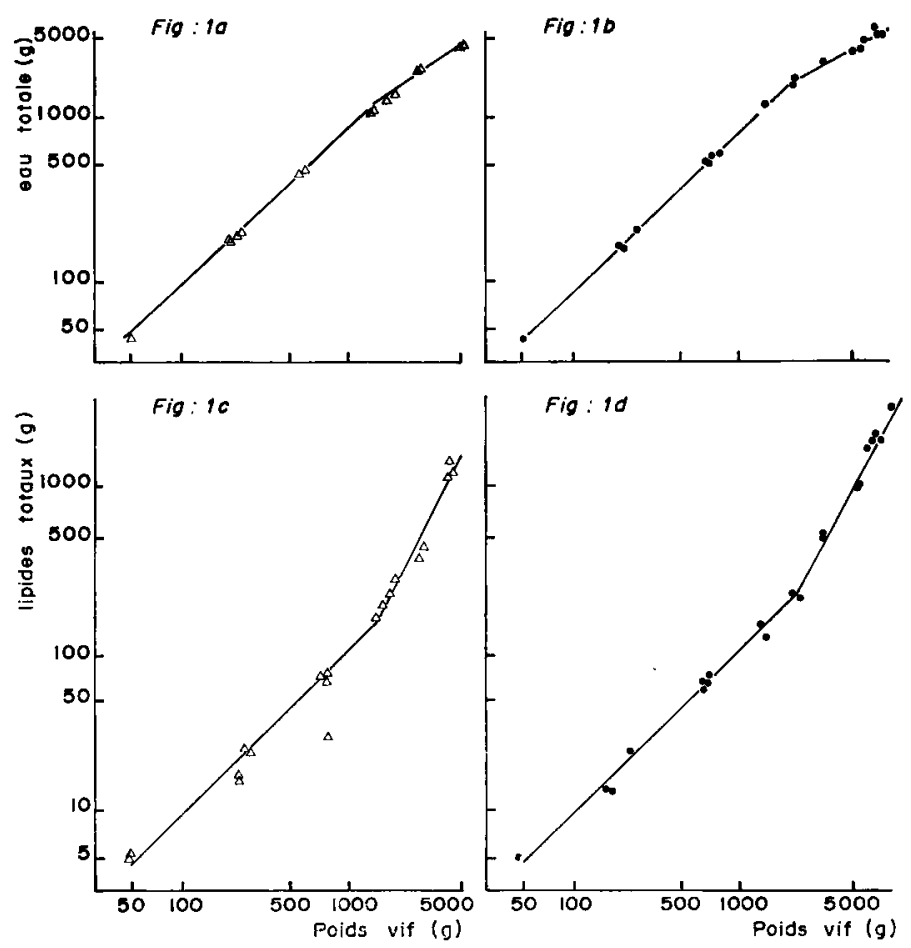

Fı. I. - Évolution relative de la quantite d'eau corporelle chez les mâles (fig. I a) et les femelles (fig. I $b$ ) ainsi que la quantité de lipides chez les mâles (fig. I $c$ ) et les femelles (fig. I $d$ ) (coordonnées logarithmiques) 


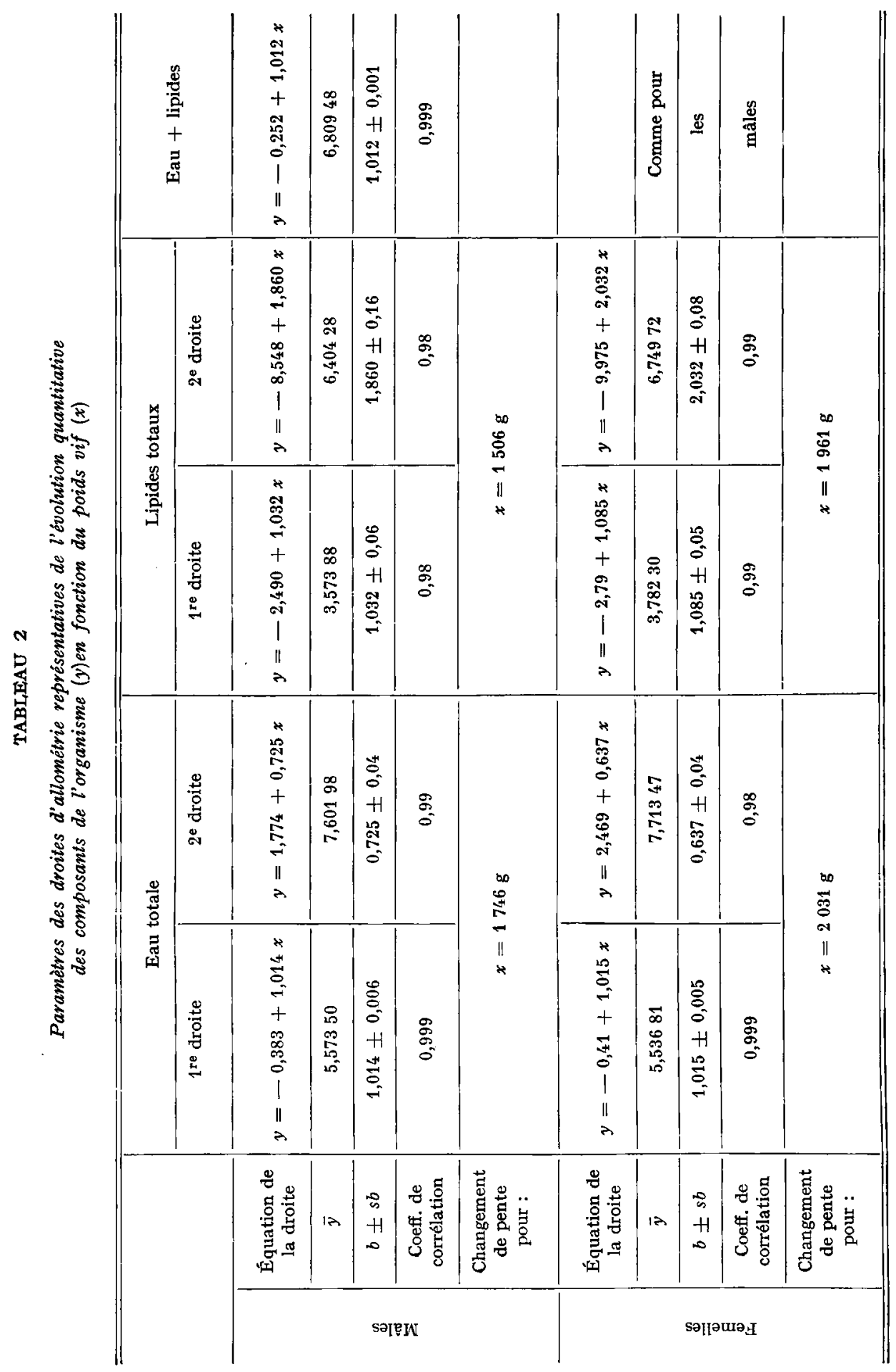


deux modalités distinctes. En deçà d'un seuil d'environ I $750 \mathrm{~g}$ de poids vif chez les mâles, $2030 \mathrm{~g}$ chez les femelles, l'augmentation de la quantité d'eau est isométrique à l'augmentation de poids vif. Au-delà elle suit une loi d'allométrie nettement minorante (fig. I $a, \mathrm{I} b$ ).

L'évolution de la quantité totale de lipides corporels se fait également selon deux phases successives d'allométrie, le passage de l'une à l'autre s'effectuant vers I $500 \mathrm{~g}$ de poids vif chez les mâles et vers I $960 \mathrm{~g}$ chez les femelles (fig. I $c$, I $d$ ).

La première est une phase d'isométrie (comme dans le cas de l'eau totale), la seconde est, par contre, une phase d'allométrie majorante.

Dans les deux sexes, CANTIER et al. (I969) ont observé pour la croissance des dépôts adipeux dissécables, une période de changement d'allométrie. Le changement d'allométrie des lipides totaux se situe pendant cette période. Nous relevons ainsi une bonne correspondance entre l'évolution des lipides totaux de l'organisme et celle des dépôts adipeux dont le développement est tardif.

Quel que soit le sexe de l'animal, l'évolution de la somme eau + lipides est isomé. trique, les variations de l'un des composants étant compensées simultanément par une variation inverse de l'autre (fig. 2).

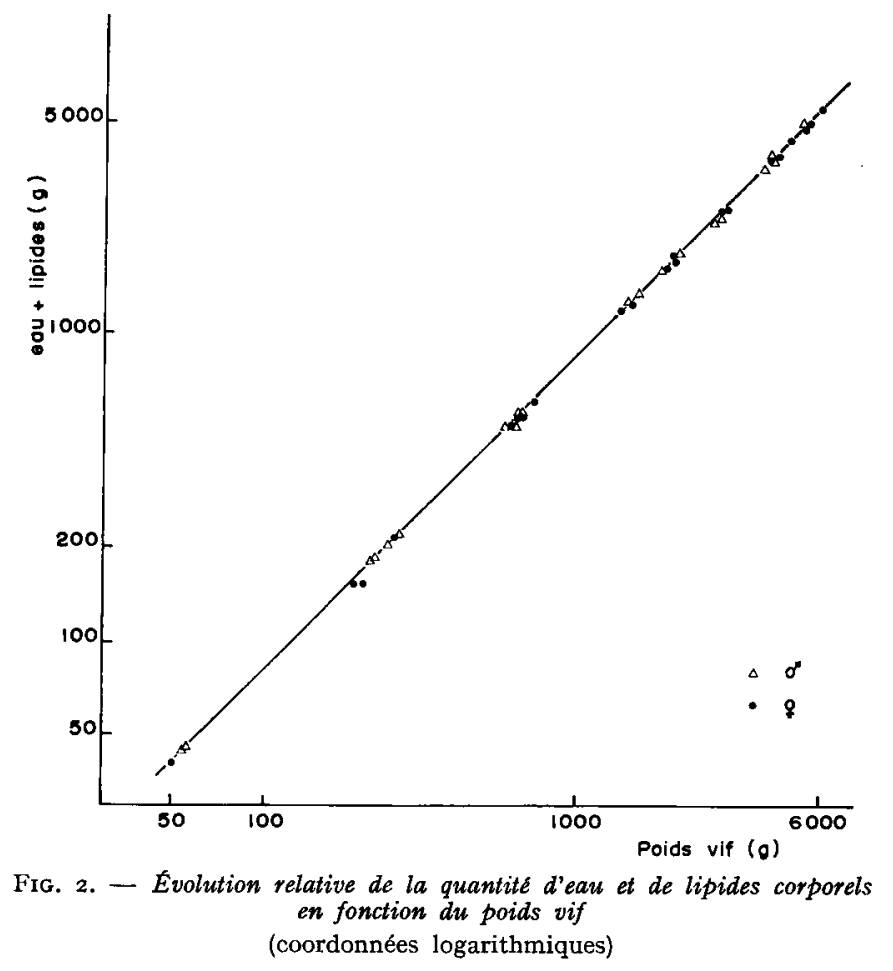

\section{2. - Évolution de l'eau et des lipides totaux du muscle Grand Psoas}

Si l'on considère la part prise par l'eau et les lipides dans la croissance pondérale du Grand Psoas, on note une allométrie légèrement minorante pour l'eau, majorante pour les lipides, la somme des deux étant isométrique au gain de poids du 
muscle (tabl. 3). Contrairement à ce qui se passe pour la composition corporelle, on n'observe pas de changement de pente pour les droites représentatives, valables quel que soit le sexe.

\section{TABLEAU 3}

Paramètres des droites d'allométrie représentatives de l'évolution quantitative des composants du muscle $(y)$ en fonction du poids vif $(x)$

\begin{tabular}{|c|c|c|c|}
\hline & Eau totale & Lipides totaux & Eau + lipides \\
\hline $\begin{array}{l}\text { Équation de } \\
\text { la droite }\end{array}$ & $y=-0,232+0,978 x$ & $y=-2,754+1,104 x$ & $y=-0,141+0,992 x$ \\
\hline $\bar{y}$ & 2,08008 & 0,03777 & 2,20451 \\
\hline$b \pm s b$ & $0,978 \pm 0,004$ & $1,104 \pm 0,002$ & $0,992 \pm 0,003$ \\
\hline $\begin{array}{l}\text { Coeff. de } \\
\text { corrélation }\end{array}$ & 0,999 & 0,996 & 0,998 \\
\hline
\end{tabular}

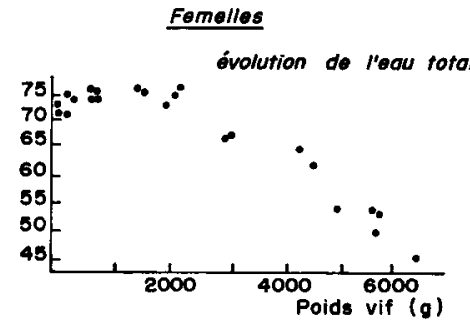

Mâles
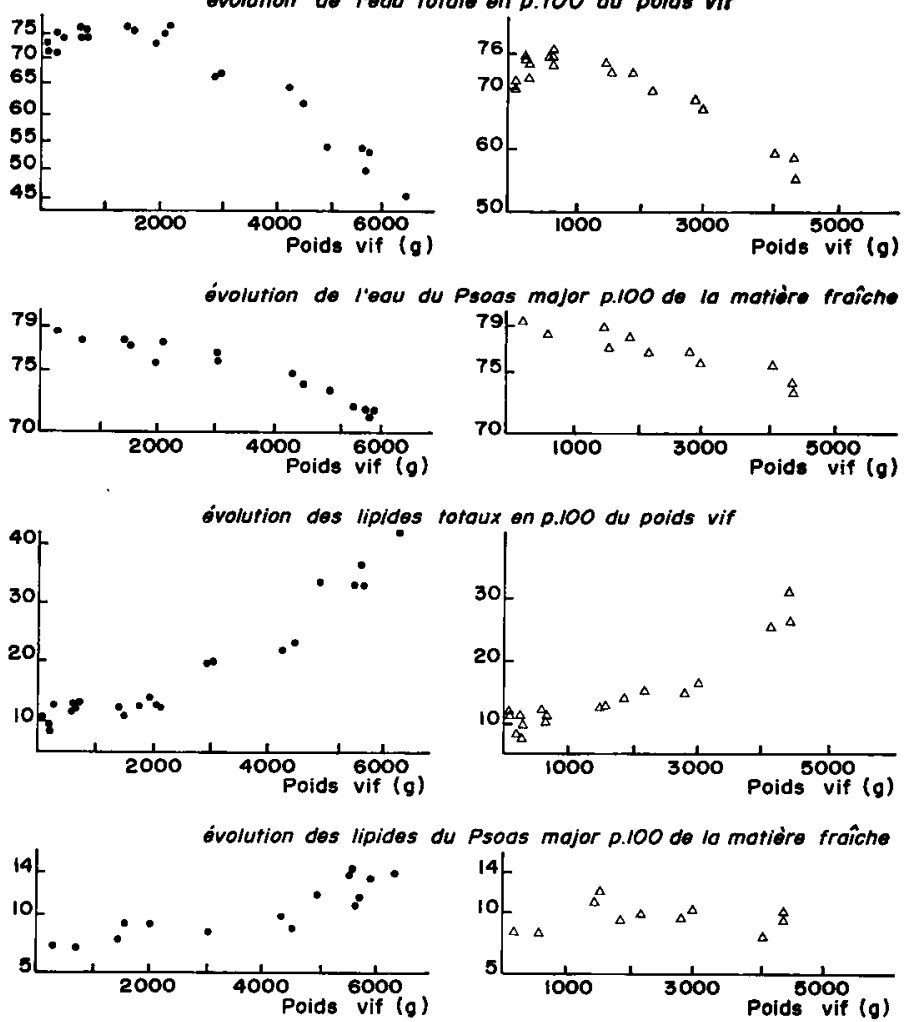

FIG. 3. - Evolution de la teneur en eau et en lipides du corps et du muscle grand psoas 


\section{DISCUSSION}

\section{Déshydratation de l'organisme.}

Au cours de la croissance postnatale, le Lapin présente donc une évolution de la composition corporelle, comparable à celle des autres mammifères. Nos résultats sont voisins de ceux de SpRAY et WIDDOWson (I950). Cependant, les animaux étudiés étant choisis au cours d'une période de vie beaucoup plus longue, nous voyons que la déshydratation relative de l'organisme est beaucoup plus importante chez les animaux les plus âgés, et qu'elle est contrebalancée par un enrichissement en lipides plus marqué. Ceci ne semble guère compatible avec les idées de MoulToN (I923), qui arrive à la conclusion que lorsque la " maturité chimique " est atteinte, vers environ 4,4 p. Ioo de 1'âge total possible, on n'observe plus que des changements mineurs dans la composition de la masse délipidée du corps.

La représentation allométrique des phénomènes permet une meilleure compréhension des mécanismes biochimiques de la croissance.

Après la naissance, deux phases sont à considérer ; pendant chacune d'elles, l'eau comme les lipides prend une part constante dans l'augmentation de poids vif, comparable dans le pretiière phase (isométrie), mais inverse et complémentaire dans la seconde (allométrie majorante pour les lipides, minorante pour l'eau). Ceci reste valable pour des animaux très gras ou à croissance fortement retardée.

Une semblable évolution avait été notée dans l'espèce humaine. Frirs-HaNSEN (I955), reprenant des résultats de diverses origines, observe un tel changement d'allométrie de l'augmentation de l'eau corporelle entre 3 et Io $\mathrm{kg}$ de poids vif.

\section{Complémentarité de l'eau et des lipides totaux de l'organisme.}

Depuis longtemps déjà, VoN HossLIN (I88I) cité par Moulton (I923) et REID et al. (I955), avaient remarqué qu'une sorte d'équilibre existe entre les lipides et l'eau, les animaux contenant d'autant moins d'eau qu'ils sont plus gras. Il en avait été déduit implicitement qu'entre l'eau et les lipides il existait une relation permettant de calculer le pourcentage de l'un, connaissant l'autre, et inversement. Une telle relation a déjà été établie chez plusieurs espèces, et en particulier chez le Lapin où elle se révèle linéaire (PANARETTO, I963).

Des formules telles que celle de Pace et Rathbun (I945),

$$
\text { Gras p. IOo }=\text { I0o }-\frac{\text { eau totale du corps p. Ioo }}{0,73^{2}}
$$

ont été déterminées, attribuant une valeur constante (73 p. Ioo environ) à la concentration d'eau dans la masse corporelle dégraissée, chez le Cobaye, le Rat, le Chat, le Chien, ce qui n'est pas conforme à la réalité, et ne tient compte ni de l'évolution de la teneur en eau tout au long de la vie, ni des importantes variations individuelles. De la même manière des coefficients voisins ont été déterminés par PANARETTo (I963) chez le Lapin : 72,6 \pm I,I, et par Ouhayoun (r969) chez la Poule 73,25 \pm 2,0y. Cette notion de masse curporelle dégraissée avait déjà été critiquée par MoORE 
et BOYDEN, 1963. En fait, après la naissance, c'est la masse globale d'eau et de lipides qui prend une même part dans le gain de poids tout au long de la vie. Des variations individuelles de la quantité d'eau et de lipides semblent d'ailleurs se compenser presque exactement, puisque la corrélation entre la somme des deux constituants et le poids vif est meilleure que celle calculée entre chacun d'eux et le poids vif. A poids vif égal, un animal plus "gras " contiendra une quantité d'eau inférieure à celle d'un animal " maigre ", la somme de l'eau et des lipides étant cependant égale dans l'un et l'autre cas.

Il en résulte que si l'on connaît le poids vif et la quantité de l'un des deux constituants, on peut déterminer la quantité de l'autre avec une bonne précision, au moins chez le Lapin.

La formule de Moulton (I923) : " l'état d'adiposité d'un individu n'a pas d'effet sur la composition estimée sur une base dégraissée " n'est donc que très approximative. Il ne sert donc à rien d'exprimer des résultats sur la base d'une composition dégraissée.

\section{Signification des changements d'allométrie.}

Le problème se pose de la signification physiologique du changement d'allométrie observé au niveau global. On peut faire plusieurs hypothèses à ce sujet.

D'une part, on peut considérer que la phase la plus active de la croissance pondérale est achevée lorsque les lapins étudiés ici atteignent un poids vif de $2 \mathrm{~kg}$. Sachant que d'une façon générale l'apport énergétique alimentaire intervient de façon importante pour la multiplication cellulaire (CHEEK et al., I965; DURAND et al., r967) et qu'à ce stade elle n'aurait plus qu'un faible rôle à jouer (CORDESSE, I970), on peut penser que cet apport sert dès lors essentiellement à la constitution des réserves adipeuses, une fois couverts les besoins d'entretien.

Il est possible également qu'une telle modification des phénomènes métaboliques soit en rapport avec une modification de l'équilibre neuro-endocrinien, le tissu adipeux, dernier à se développer dans l'organisme, prenant alors une part importante dans la croissance pondérale, la contribution de l'eau étant diminuée d'autant.

YANNET et DARRow ont montré chez le Chat (1938) que parmi les secteurs liquidiens de 1'organisme, la concentration de l'eau intracellulaire varie peu au cours de la vie. Nous voyons ici que la teneur en eau du muscle varie moins que celle de 1'organisme. Chez le Lapin, c'est donc probablement en rapport avec une diminution relative de l'eau extracellulaire que se fait le développement du tissu adipeux, après ce poids vif de $2 \mathrm{~kg}$ environ.

\section{CONCLUSION}

Nous voyons ainsi que, chez le Lapin après la naissance, une augmentation de poids s'accompagne toujours d'un changement de composition corporelle. Mais, quelles que soient les variations individuelles dans le degré d'adiposité, l'eau et les lipides totaux se comportent ensemble comme un secteur unique de l'organisme, prenant une part constante dans la croissance pondérale. Cette propriété, ainsi systématisée, semble d'un intérêt pratique considérable. En effet, connaissant le poids vif de l'animal et la quantité d'eau contenue dans l'organisme, on pourra déterminer 
la quantité de lipides présente chez cet animal, même vivant, quels que soient son âge et son poids. Cela semble pouvoir contribuer à faciliter les recherches d'ordre nutritionnel.

Rę̧u pour publication en avril 1971.

\section{SUMMARY}

\section{POSTNATAL, CHANGES IN WATER AND LIPID AMOUNTS}

OF THE WHOLE BODY AND THE PSOAS MAJOR MUSCLE, IN THE RABBIT

Postnatal changes in the biochemical composition of the whole body and the psoas major muscle were investigated on male and female rabbits.

Total lipids were estimated from body and muscle homogenates by Folch's technique. Total water was estimated by vaccum desiccation at $60^{\circ} \mathrm{C}$.

The composition of the psoas major muscle fluctuated less than that of the whole body irrespective of sex (fig. 3).

The relative growths of total water and lipid amounts of the body was investigated using Huxley's allometry equation. In both sexes, after an isometric period, there was a simultaneous allometry change, accelerating for the lipids and decelerating for the water from I $75^{\circ}$ and $2030 \mathrm{gm}$. body weight for the male and the female respectively. After this change, the part played by lipids in body weight gain was higher in the female (slope 2.03) than in the male (slope r.86) (table 2).

The (water + lipids) amount increase and body growth were isometric for the whole postnatal life irrespective of sex logarithmic plotting was as follows :

$\begin{aligned} y & =0.252+\text { r.o1 } 2 x \\ \text { Where } y & =\log \text { (water }+ \text { lipids) and } x=\log \text { (live weight) }\end{aligned}$

It is therefore possible, given the amount of water and the body live weight, to predict the amount of lipids, regardless of the age or weight of rabbits.

\section{RÉFÉRENCES BIBLIOGRAPHIQUES}

Allen T. H., Welch B. E., Trujillo T. T., Roberts J. E., I959. Fat, water and tissue solids of the whole body less its bone mineral. J. appl. Physiol., 14, 1009-1012.

Baron R., Vigneron P., I97o. Hétérogonie biochimique des composants azotés du grand psoas au cours de la croissance postnatale du lapin. Ann. Biol. anim. Biochim. Biophys., 10, 223-238.

Baron R., Vezinhet A., Cantier J., r97o. Allométrie de croissance chez le Lapin. II. Thymus Ann. Biol. anim. Biochim. Biophys. (sous presse).

BEzold von A., I857. Untersuchungen über die Verteilung von Wasser, organischer Materia und anorganischer Verbindungen im Tierreiche. Z. Wiss. Zool., 8, 487. Cité par C. R. Moulton, I923.

Cantier J., Vezinhet A., Rouvier R., Dauzier L., ig69. Allométrie de croissance chez le Lapin (Oryctolagus cuniculus). I. Principaux organes et tissus. Ann. Biol. anim. Biochim. Biophys., 9, 5-39.

CheEk D. B., Holt A. B., I963. Growth and body composition of the mouse. Amer. J. Physiol., 205, 9 I3-918.

Chenk D. B., Powell G. K., Scott R. E., I965. Growth of muscle mass and skeletal collagen in the rat. I. Normal growth. II. The effect of ablation of pituitary, thyroid or testes. Bull. Johns Hopkins Hosp., 116, 378-395.

CORDESSE R., I97o. Communication personnelle.

Dukes H. H., 1947. The physiology of domestic animals. Comstock Publishing Co : Ithaca, New York.

Durand G., Fauconneau G., Penot E., I967. Croissance des tissus du rat et réduction de l'apport énergétique de la ration; influence sur la teneur en acides nucléiques. Ann. Biol. anim. Biochim. Biophys 7 , $145-155$.

Ellenberger H. B., Newlander J. A., Jones C. H., i95o. Composition of the bodies of dairy cattle. Vt. Agr. Expt. Stn. Bull., 558. 
Folch J., Lees M., Sloane-Stanley G. H. S., 1957. A simple method for the isolation and purification of total lipids from animal tissues. J. biol. Chem, 226, 497-509.

Frirs-Hansen B., t955. Changes in body water compartments during growth. Acta paedia. Stockh., 46, suppl. I09, I-67.

Gordon E. E., Kowalski K., Fritss M., rg66. Muscle proteins and DNA in rat quadriceps during growth. Am-. J. Physiol., 210, 1033-1040.

Guillaume J., Pion R., de Reviers M., Delpech P. x966. Discontinuité de croissance de divers constituants corporels chez le jeune poulet. C. R. Acad. Sci. Paris, 262, 2506-2509.

Helander E., 1959. Fat content of skeletal muscular tissue. Acta Morphol. Res. Scand., 2, $230-254$.

Hossuin von R., I88I. Über dem Wasser und Fettgehalt der Organe bei verschiedenen pathologischen Zustanden. Inaugural dissertion, 3 U., 4, München r88I. Cité par C. R. Moultow, I923.

HuXLEY J. S., 1932. Problems of relative growth. 276 p., Methuen, London.

Ivanov I. I., r967, Organic substances of the striated muscle. Symp. Biol. Hung., 8, 89-124.

Kermack K. A., Haldane J. B. S., I950. Organic correlation and allometry. Biometrika, 37, 40-4I.

Moore F. D., Boyden C. M., r963. Body cell mass and limits of hydration of the fat-free body : their relation to estimated skeletal weight. Ann. N. Y. Acad. Sci., 110, 62-7r.

Moulton C. R., r923. Age and chemical development in mammals. J. biol. Chem., 57, 79-97.

Moulton C. R., Trowbridge P. F., Haigh L. D., 1922. Studies in animal nutrition. III. Changes in chemical composition on different planes of nutrition. Bull. Mo. agric. Exp. Stn, 55.

NeEDhaM J., 1934. Chemical heterogony and the ground plan of animal growth. Biol. Rev., 9, 79-10g.

OUHAYOUN J., I970. Estimation des lipides corporels du poulet par la méthode de dilution isotopique de l'eau tritiée. Ann. Biol. anim. Biochim. Biophys., 10, 621-630.

Pace N., Rathbun E. N., 1945. Studies on body composition. III. The body water and chemically combined nitrogen content in relation to fat content. J. biol. Chem., 158, 685-69 I.

Panaretro B. A., 1963. Body composition in vivo. I. The estimation of total body water with antipyrine and the relation of total body water to total body fat in rabbits. A ust. J. agric. Res., 14, 594-6or.

Panaretro B. A., TILl A. R., I 963 . Body composition in vivo. II. The composition of mature goats and its relationships to the antipyrine tritiated water and $\mathrm{N}$-acetyl 4 -amidoantipyrine spaces. $A$ ust. $J$. agric. Res., 14, 926-944.

Panaretto B. A., 1963 . Body composition in vivo. III. The composition of living ruminants and its relation to the tritiated water spaces. Aust. J. agric. Res., 14, 944-952.

Prud'hon M., Vezinhet A., Cantier J., r969. Croissance, qualités bouchères et coût de la production des lapins de chair. Bull. tech. Inf. Ingrs. Servs agric., 248, 203-2I2.

Reid J. T., Wellington G. H., DunN H. O., I955. Some relationships among the major chemical components of the bovine body and their application to nutritional investigations. J. Dairy. Sci., 88, I344-I359.

Spray C. M., Widdowson E. M., I950. The effect of growth and development on the composition of mammals. Br. J. Nutr., 4, 332-353.

Terssier G., 1934. Dysharmonies et discontinuités dans la croissance. Actualités scientifiques et industrielles, 95, Hermann. Paris, pp. 39.

Vezinhet A., Rouvier R., Dulor J. P., Cantier J., r97r. Allométrie de croissance chez le Lapin. III. Principales régions des systèmes musculaire et osseux (sous presse).

YANNET H., DARROW D. C., 1938. The effect of growth on the distribution of water and electrolytes in brain, liver and muscle. J. biol. Chem., 128, 295-305. 